\title{
T-duality off shell in 3D type II superspace
}

\section{Martin Poláček and Warren Siegel ${ }^{1}$}

C.N. Yang Institute for Theoretical Physics, State University of New York, Stony Brook, NY 11794-3840, U.S.A.

E-mail: martin.polacek@stonybrook.edu, siegel@insti.physics.sunysb.edu

ABSTRACT: We give the manifestly T-dual formulation of the massless sector of the classical 3D Type II superstring in off-shell $3 \mathrm{D} \mathcal{N}=2$ superspace, including the action. It has a simple relation to the known superspace of $4 \mathrm{D} \mathcal{N}=1$ supergravity in $4 \mathrm{D}$ M-theory via $5 \mathrm{D}$ F-theory. The prepotential appears as part of the vielbein, without derivatives.

KeYwords: Supersymmetry and Duality, Superspaces, Supergravity Models, F-Theory

ARXiv EPRINT: 1403.6904

\footnotetext{
${ }^{1}$ http://insti.physics.sunysb.edu/ siegel/plan.html
} 


\section{Contents}

1 Introduction 1

2 F-theory (membrane vs. strings) 2

2.1 F-theory and its compactification 2

$2.25 \mathrm{D}$ vs. $4 \mathrm{D}$ vs. $3 \mathrm{D}$ - Compactifications 3

3 Algebra 4

3.1 Current algebra of $Z_{\mathcal{M}}$

$\begin{array}{lll}3.2 & \text { Background fields } & 6\end{array}$

$\begin{array}{lll}3.3 & \text { Further constraints and gauge fixing } & 7\end{array}$

3.4 Dimensional constraints: solution 8

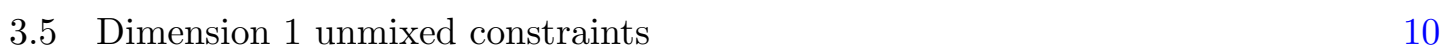

$\begin{array}{ll}3.6 & \text { Dimension } 1 \text { mixed constraints } \\ & 11\end{array}$

$\begin{array}{lll}3.7 & \tilde{T}=0 & 12\end{array}$

4 Cartan-Killing metric and field equations $\quad 13$

$\begin{array}{lll}4.1 & \text { Cartan-Killing metric } & 13\end{array}$

$\begin{array}{lll}4.2 & \text { Field equations } & 14\end{array}$

$\begin{array}{ll}4.3 \text { Field equations: summary } & 16\end{array}$

$\begin{array}{lll}5 & \text { Dilaton } & 17\end{array}$

$\begin{array}{llr}6 & \text { Conclusion } & 17\end{array}$

\section{Introduction}

In paper [1] we obtained the curvature tensor (previously discovered in [2-4]) in a way manifestly covariant under $\mathrm{O}(\mathrm{D}, \mathrm{D}) \mathrm{T}$-duality. The aim of this paper is to extend the techniques of the T-dually extended spaces from the bosonic case to the supersymmetric case. In $\mathrm{D}=10$, this would simultaneously describe Types IIA and IIB supergravity; here we consider the simpler case of $\mathrm{D}=3$, where the theory can easily be treated off shell.

Manifest T-duality is in general constructed by doubling the space-time coordinates, as shown in [2-4]. This doubled space-time is further extended by the coordinates for the Lorentz generators. The dependence of the background vielbein on them is fully fixed (up to gauge) by the coset constraints [2-4]. This is done by requiring that the associate torsions take their vacuum values. The generalisation of this approach to string theory requires the use of the affine symmetry algebra (oscillator algebra together with the Lorentz algebra). The consistency (closure of the Jacobi identities) of the new affine symmetry algebra requires addition of the new current $\Sigma^{m n}$ for every Lorentz current $S_{m n}$. We also 
add the new coordinates for the current $\Sigma^{m n}$. (The necessity of this new current was first realized in the context of $\mathrm{AdS}_{5} \times \mathrm{S}_{5}[5,6]$.) In the supersymmetric case the fermionic coordinates are doubled as well. For the fermionic current $D_{\mu}$ we need to add a dual current $\Omega^{\mu}$ (for the consistency of the affine supersymmetry algebra), see [2-4]. By this way we obtain the affine supersymmetry algebra with the extra currents $\Omega^{\mu}, \Sigma^{m n}$.

The generalized torsion is constructed from this affine Lie algebra in a general background, which acts as the stringy generalization of covariant derivatives. Because of the additional currents, the enlarged vielbein that describes this background includes the Lorentz connection, and the enlarged torsion includes also the curvature. Closure of the algebra implies the orthogonality constraints $E \eta E^{T}=\eta$ on the vielbein. In this paper we solve these just at the linear level together with the coset constraints also solved at linear level. There is also an extension of dimensional reduction to the usual D coordinates.

In this article (as a starting point for a bigger program on T-dually extended superspaces) we consider the 3 dimensional T-dually extended superspace. The higher dimensional case is discussed in [7]. We would also see that this (toy) model of 3 dimensional T-dually extended space goes with the idea of lower dimensional F-theory (i.e., the lower dimensional analogue of 12 dimensional F-theory, see [8]). At the end we will show that the physical spectrum (and the structure) of the theory coincides with $\mathcal{N}=2$ supergravity in 3 dimensions (after dimensional reduction). That should be expected since as we will show classical $\mathcal{N}=1$ supergravity in 4 dimensions can be interpreted as to have the same F-theory origin as (manifestly) T-dual (covariant) 3D supergravity. So does 3D $\mathcal{N}=2$ supergravity (after compactification of $4 \mathrm{D} \mathcal{N}=1$ supergravity to $3 \mathrm{D}$ ). The doubling in this paper is obtained naturally from compactification of F-theory along one space direction. We will get: $S O(3,2) \rightarrow S O(2,2) \simeq S O(2,1) \otimes S O(2,1)$ (to be explained in the text), which is $\mathrm{T}$-dual $\mathcal{N}=2$ string theory and effectively T-dual $3 \mathrm{D} \mathcal{N}=2$ supergravity.

We follow the procedure described in articles $[1-4,7]$. The differences are that we work just to linear order in fields and in 3D T-dual superspace. On top of that we will also find the relation of the T-dually extended theory to the (lower dimensional analog of) F-theory.

\section{F-theory (membrane vs. strings)}

\section{$2.1 \quad$ F-theory and its compactification}

F-theory was first proposed by Cumrun Vafa as 12 dimensional theory, see [8]. The theory is further compactified on the two-torus or more generally on elliptically fibered Calabi-Yau manifolds. We discuss the 5 dimensional analogue of this theory. We want to motivate the natural identification between $4 \mathrm{D} \mathcal{N}=1$ supergravity, further compactified to $3 \mathrm{D} \mathcal{N}=2$ $(3 \mathrm{D} \mathcal{N}=2$ supergravity is recently discussed in [9-11]), and T-dual $3 \mathrm{D} \mathcal{N}=2$ string theory. Both can be thought to have an origin in higher dimensional F-theory. This theory will be further compactified in two ways. One compactification produces 4 dimensional M-theory that will effectively become $\mathcal{N}=1$ supergravity with the specific chiral compensator that contains a 3 -form. This is expected since this $\mathcal{N}=1$ supergravity is an effective theory of 2-branes (discussion of the lower dimensional supersymmetric membrane theory can be 
found in [12], (super) membrane theory discussed in [13-16]). The other compactification gives 3 dimensional T-dual $\mathcal{N}=2$ string theory, so effectively T-dual $\mathcal{N}=2$ supergravity.

\section{$2.25 \mathrm{D}$ vs. $4 \mathrm{D}$ vs. $3 \mathrm{D}-$ Compactifications}

5 dimensional F-theory is the (supersymmetric) 2-brane theory in the space with signature $(+,+,+,-,-)$. The Lorentz group is $S O(3,2)$. We can pick the physical time direction and compactify F-theory along the other time direction, so we will get the Lorentz group breaking $S O(3,2) \rightarrow \mathrm{SO}(3,1)$. The 4 dimensional $\mathcal{N}=1 \mathrm{SO}(3,1)$ theory is just 4 dimensional M-theory, which is effectively 4 dimensional $\mathcal{N}=1$ supergravity. We can also pick one space direction and compactify F-theory along this direction, so we will get: $S O(3,2) \rightarrow S O(2,2) \simeq S O(2,1) \otimes S O(2,1)$, which will become T-dual $\mathcal{N}=2$ string theory and effectively T-dual $3 \mathrm{D} \mathcal{N}=2$ supergravity. If we further compactify 4 dimensional $\mathcal{N}=1$ supergravity along a space direction we will get 3 dimensional $\mathcal{N}=2$ supergravity coupled to a vector multiplet. On the other hand, if we take T-dual $3 \mathrm{D} \mathcal{N}=2$ theory and compactify half of the (doubled) dimensions we would again get $3 \mathrm{D} \mathcal{N}=2$ supergravity coupled to a vector multiplet. We therefore have the natural identification of the objects from $4 \mathrm{D} \mathcal{N}=1$ supergravity (further compactified) and T-dual $3 \mathrm{D} \mathcal{N}=2$ supergravity. We can therefore use the techniques of T-dually extended superspace and derive $3 \mathrm{D} \mathcal{N}=2$ supergravity coupled to a vector multiplet.

In $4 \mathrm{D}\left(n=-\frac{1}{3}\right.$ minimal and linearised) supergravity we have the prepotential $H_{\alpha \dot{\beta}}$ and the scalar prepotential $\mathcal{V}$. The scalar prepotential becomes a particular (chiral) compensator of the form $\phi=\bar{D}^{2} \mathcal{V}$. That contains a 3-form, see section 4.4.d in [17], or more generally [18]. This is expected since $4 \mathrm{D} \mathcal{N}=1$ supergravity is the effective theory for 2-branes.

The $4 \mathrm{D} \mathcal{N}=1$ gauge transformations are, see section 5.2 in $[17,19]$ :

$$
\delta H_{\alpha \dot{\beta}}=D_{\alpha} \bar{L}_{\dot{\beta}}-\bar{D}_{\dot{\beta}} L_{\alpha} \text { and } \delta \mathcal{V}=D^{\alpha} L_{\alpha}+\bar{D}^{\dot{\alpha}} \bar{L}_{\dot{\alpha}}
$$

where $D_{\alpha}$ and $\bar{D}_{\dot{\alpha}}$ are usual $4 \mathrm{D} \mathcal{N}=1$ covariant derivatives. We can dimensionally reduce the theory to $3 \mathrm{D}$ and obtain the $3 \mathrm{D} \mathcal{N}=2$ theory. Using dimensional reduction we get:

$$
D_{\alpha}=\frac{1}{\sqrt{2}}\left(D_{\alpha}+i D_{\alpha^{\prime}}\right) \text { and } \bar{D}_{\dot{\alpha}}=\frac{1}{\sqrt{2}}\left(D_{\alpha}-i D_{\alpha^{\prime}}\right)
$$

where $D_{\alpha}$ and $D_{\alpha^{\prime}}$ are real $3 \mathrm{D} \mathcal{N}=2$ covariant derivatives. The gauge parameters can be written as:

$$
L_{\alpha}=\frac{1}{\sqrt{2}}\left(\Lambda_{\alpha}-i \Lambda_{\alpha^{\prime}}\right) \text { and } \bar{L}_{\dot{\alpha}}=\frac{1}{\sqrt{2}}\left(\Lambda_{\alpha}+i \Lambda_{\alpha^{\prime}}\right) .
$$

The $3 \mathrm{D} \mathcal{N}=2$ gauge transformations thus are:

$$
\begin{aligned}
\delta H_{(\alpha \dot{\beta})} & =\delta H_{\alpha \beta^{\prime}}=i\left(D_{\left(\alpha^{\prime}\right.} \Lambda_{\beta)}+D_{(\alpha} \Lambda_{\left.\beta^{\prime}\right)}\right) \\
\delta H_{[\alpha \dot{\beta}]} & =\delta V=D^{\alpha} \Lambda_{\alpha}-D^{\alpha^{\prime}} \Lambda_{\alpha^{\prime}} \\
\delta \mathcal{V} & =D^{\alpha} \Lambda_{\alpha}+D^{\alpha^{\prime}} \Lambda_{\alpha^{\prime}}
\end{aligned}
$$




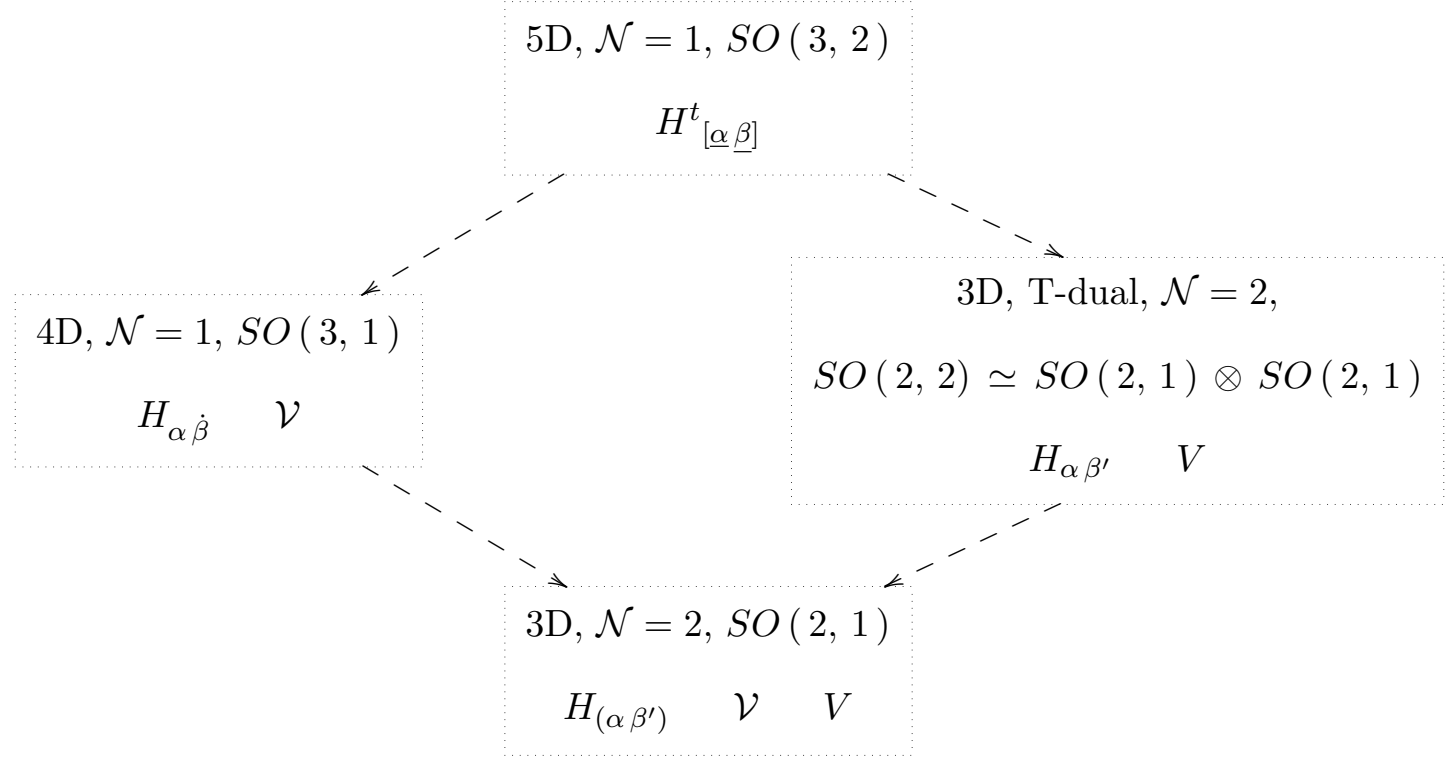

Figure 1. F-theory breaking.

The $4 \mathrm{D} \mathcal{N}=1$ prepotential $H_{\alpha \dot{\beta}} \equiv\left(H_{(\alpha \dot{\beta})}, H_{[\alpha \dot{\beta}]}\right)$ is a $4 \mathrm{D}$ vector and becomes the $3 \mathrm{D}$ vector $H_{\left(\alpha \beta^{\prime}\right)}$ and a prepotential $V$ (for a vector multiplet). We also have the 4D prepotential $\mathcal{V}$ (for the chiral compensator $\phi=\bar{D}^{2} \mathcal{V}$ ) that becomes the 3D prepotential $\mathcal{V}$. On the other hand the 3D T-dual prepotential (symmetric part) $H_{\left(\alpha \beta^{\prime}\right)}$ (after dimensional reduction to $3 \mathrm{D} \mathcal{N}=2$ ) is again a vector (describing conformal supergravity) but $H_{\left[\alpha \beta^{\prime}\right]}$ becomes the prepotential $\mathcal{V}$, see the transformations (3.19), and the prepotential $H_{\alpha \beta^{\prime}}$ is just part of the vielbein, see table 3 . Finally the $3 \mathrm{D}$ T-dual $\mathcal{N}=2$ prepotential $V$ becomes the prepotential for the vector multiplet in $3 \mathrm{D} \mathcal{N}=2$ supergravity.

Therefore we have an identification between $3 \mathrm{D} \mathcal{N}=2$ T-dual supergravity and $3 \mathrm{D} \mathcal{N}=2$ supergravity coupled to a vector multiplet: $H_{\left(\alpha \beta^{\prime}\right)} \rightarrow H_{\left(\alpha \beta^{\prime}\right)}, H_{\left[\alpha \beta^{\prime}\right]} \rightarrow \mathcal{V}$ and $V \rightarrow V$.

We also have the identification between $4 \mathrm{D} \mathcal{N}=1$ supergravity and $3 \mathrm{D} \mathcal{N}=2$ supergravity coupled to a vector multiplet: $H_{(\alpha \dot{\beta})} \rightarrow H_{\left(\alpha \beta^{\prime}\right)}, H_{[\alpha \dot{\beta}]} \rightarrow V$ and $\mathcal{V} \rightarrow \mathcal{V}$.

The situation is summarised in the above diagram 1 , where $\left.H_{[\underline{\alpha}}^{t} \underline{\beta}\right]$ is the 5 dimensional prepotential ( $\underline{\alpha} \in\{1, \ldots 4\}$, " $t$ " means that it is traceless, it has 5 real components).

\section{Algebra}

We give a very brief outline of the algebraic objects and steps that will lead to the formulation of linearised T-dual 3D supergravity. The interested reader may see references [1-4] (where the subject is explained in great detail).

\subsection{Current algebra of $Z_{\mathcal{M}}$}

As in paper [1], we consider the (super)string generalisation of the string oscillator algebra. Because of T-duality and the (super)Bianchi identity the current algebra has a structure:

$$
\left[Z_{\mathcal{M}}(1), Z_{\mathcal{N}}(2)\right]=-i \eta_{\mathcal{M N}} \delta^{\prime}(2-1)-i f_{\mathcal{M N}}{ }^{\mathcal{P}} Z_{\mathcal{P}} \delta(2-1)
$$


where $Z_{\mathcal{M}}:=\left(S_{M N}, D_{\mu}, P_{M}, \Omega^{\mu}, \Sigma^{M N}\right)$ is the generalisation of the (super)string oscillators and the metric $\eta_{\mathcal{M N}}$ (given later). The $P_{M}$ generators are the $O(D, D)$ generalisation of string oscillators $P_{m}$. In an explicit $O(D, D)$ basis the $P_{M}$ generators are given as: $P_{M}:=\left(P_{m}, X^{\prime m}\right)$. For future purposes we want to use a different, left/right basis. In the left/right basis $P_{M}:=\left(P_{\mathbf{m}}, P_{\tilde{\mathbf{m}}}\right)=\frac{1}{\sqrt{2}}\left(P_{m}+X_{m}^{\prime}, P_{m}-X_{m}^{\prime}\right)$. The Lorentz generators also have the left/right structure: $S_{M N}:=\left(S_{\mathbf{m n}}, S_{\tilde{\mathbf{m}} \tilde{\mathbf{n}}}\right)$, where $S_{\mathbf{m n}}$ are generators of left (or equivalently $S_{\tilde{\mathbf{m}} \tilde{\mathbf{n}}}$ right) Lorentz transformations. The $D_{\mu}:=\left(D_{\mu}, D_{\tilde{\mu}}\right)$ are the generators of left and right supersymmetry transformations. The generators $\Omega^{\mu}:=\left(\Omega^{\mu}, \Omega^{\tilde{\mu}}\right)$ and $\Sigma^{M N}:=\left(\Sigma^{\mathbf{m n}}, \Sigma^{\tilde{\mathbf{m}} \tilde{\mathbf{n}}}\right)$ are the new generators, needed to satisfy the Bianchi identity. For further reference see [1-4].

The full current algebra of $Z_{\mathcal{M}}$ oscillators (3.1) is the affine (super)Lie algebra (3.2) and its explicit form is:

$$
\begin{aligned}
& {\left[S_{\mathbf{m n}}(1), S_{\mathbf{k} \mathbf{l}}(2)\right]=-i \eta_{[\mathbf{m}[\mathbf{k}} S_{\mathbf{l}] \mathbf{n}]} \delta(2-1)} \\
& {\left[S_{\mathbf{m n}}(1), D_{\rho}(2)\right]=-i \frac{1}{2}\left(\gamma_{\mathbf{m n}}\right)_{\rho}^{\sigma} D_{\sigma} \delta(2-1)} \\
& {\left[S_{\mathbf{m n}}(1), P_{\mathbf{k}}(2)\right]=i \eta_{\mathbf{k}[\mathbf{m}} P_{\mathbf{n}]} \delta(2-1)} \\
& {\left[S_{\mathbf{m n}}(1), \Omega^{\rho}(2)\right]=-i \frac{1}{2}\left(\gamma_{\mathbf{m n}}\right)_{\sigma}^{\rho} \Omega^{\sigma} \delta(2-1)} \\
& {\left[S_{\mathbf{m n}}(1), \Sigma^{\mathbf{k l}}(2)\right]=-i \delta_{\mathbf{m n}}{ }^{\mathbf{k l}} \delta^{\prime}(2-1)-i \delta_{[\mathbf{m}}{ }^{[\mathbf{k}} \eta_{\mathbf{n}] \mathbf{s}} \Sigma^{\mathrm{l}] \mathbf{s}} \delta(2-1)} \\
& \left\{D_{\rho}(1), D_{\sigma}(2)\right\}=i 2\left(\gamma^{\mathbf{m}}\right)_{\rho \sigma} P_{\mathbf{m}} \delta(2-1) \\
& {\left[D_{\rho}(1), P_{\mathbf{m}}(2)\right]=-i 2\left(\gamma_{\mathbf{m}}\right)_{\rho \sigma} \Omega^{\sigma} \delta(2-1)} \\
& \left\{D_{\rho}(1), \Omega^{\sigma}(2)\right\}=-i \delta_{\rho}^{\sigma} \delta^{\prime}(2-1)-i \frac{1}{2}\left(\gamma_{\mathbf{m n}}\right)_{\rho}^{\sigma} \Sigma^{\mathbf{m n}} \delta(2-1) \\
& {\left[D_{\rho}(1), \Sigma^{\mathbf{m n}}(2)\right]=0} \\
& {\left[P_{\mathbf{m}}(1), P_{\mathbf{n}}(2)\right]=-i \eta_{\mathbf{m n}} \delta^{\prime}(2-1)+i \eta_{\mathbf{m}[\mathbf{h}} \eta_{\mathbf{n} \mid \mathbf{s}]} \Sigma^{\mathbf{h s}} \delta(2-1)} \\
& {\left[P_{\mathbf{m}}(1), \Omega^{\rho}(2)\right]=0} \\
& {\left[P_{\mathbf{m}}(1), \Sigma^{\mathbf{k l}}(2)\right]=0} \\
& \left\{\Omega^{\rho}(1), \Omega^{\sigma}(2)\right\}=0 \\
& {\left[\Omega^{\rho}(1), \Sigma^{\mathbf{m n}}(2)\right]=0} \\
& {\left[\Sigma^{\mathbf{m n}}(1), \Sigma^{\mathbf{k} \mathbf{l}}(2)\right]=0} \\
& \rightarrow \text { Same for Left } \rightarrow \text { Right } \\
& {[\text { Left, Right }\}=0}
\end{aligned}
$$

The only nonvanishing terms in the metric and structure constants are (as can be 
guessed by dimensional analysis)

$$
\eta_{P P}, \eta_{S \Sigma}, \eta_{D \Omega}, ; f_{S P P}, f_{S S \Sigma}, f_{D D P}, f_{S D \Omega}
$$

where we have lowered the upper index on $f$ with $\eta$ to take advantage of its total (graded) antisymmetry, and used "schematic" notation, replacing explicit indices with their type:

$$
\mathcal{M}:=\left({ }_{M N},{ }_{\mu},{ }_{M},{ }^{\mu},{ }^{M N}\right):=(S, D, P, \Omega, \Sigma)
$$

Explicitly these are, for the left-handed algebra,

$$
\begin{array}{rlrl}
(\eta)_{\mathbf{m n}} & =\eta_{\mathbf{m n}}, & (\eta)_{\mathbf{m} \mathbf{m}}{ }^{\mathbf{p q}}=\delta_{\mathbf{m} \mathbf{m}}{ }^{\mathbf{p q}}, & (\eta)_{\sigma}{ }^{\rho}=\delta_{\sigma}^{\rho} \\
f_{\mathbf{m} \mathbf{n}}{ }^{\mathbf{p q}}=-\delta_{\mathbf{m} \mathbf{n}}{ }^{\mathbf{p q}}, & f_{\mathbf{m n} \mathbf{p q}}{ }^{\mathbf{r s}}=\eta_{\left[\mathbf { m } \left[\mathbf{p} \delta_{\mathbf{q}] \mathbf{n}]}{ }^{\mathbf{r s}},\right.\right.} \\
f_{\sigma \rho}{ }^{\mathbf{m}}=2\left(\gamma^{\mathbf{m}}\right)_{\sigma \rho}, & f_{\mathbf{m n} \sigma^{\rho}}=-\frac{1}{2}\left(\gamma_{\mathbf{m n}}\right)_{\sigma}^{\rho}
\end{array}
$$

For the right-handed algebra we change the signs of the corresponding terms in $\eta_{\mathcal{M N}}$ but not in $f$.

For dealing with antisymmetric pairs of indices we have introduced an implicit metric such that for any two antisymmetric tensors we have

$$
A \cdot B \equiv \frac{1}{2} A^{\mathbf{m n}} B_{\mathbf{m n}}
$$

The identity matrix with respect to this inner product is

$$
\delta_{\mathbf{m n}}{ }^{\mathbf{p q}} \equiv \delta_{[\mathbf{m}}^{\mathbf{p}} \delta_{\mathbf{n}]}^{\mathbf{q}}
$$

\subsection{Background fields}

The aim is to find the linearised formulation of the 3D T-dual theory. We are following the approach used in the previous paper, see [1], section 1.2. We will briefly mention the outline here:

We want to use the T-dual formulation of the stringy generalisation of the oscillator algebra (3.2). We introduce background fields via vielbeins. Following [2-4] but using algebra (3.1) we get:

$$
\Pi_{\mathcal{A}}(1)=E_{\mathcal{A}}^{\mathcal{M}}\left(X^{\mathcal{N}}\right) Z_{\mathcal{M}}
$$

The affine Lie algebra for the $\Pi_{\mathcal{A}}$ can be compactly written as:

$$
\left[\Pi_{\mathcal{A}}(1), \Pi_{\mathcal{C}}(2)\right] \equiv-i \eta_{\mathcal{A C}} \delta^{\prime}(2-1)-i T_{\mathcal{A C}} \mathcal{E}_{\Pi_{\mathcal{E}}} \delta(2-1)
$$

where $T_{\mathcal{A C}} \mathcal{E}$ is a (super)stringy generalisation of torsion, see [1]:

$$
\begin{aligned}
& T_{\mathcal{A C}}{ }^{\mathcal{E}}=E_{[\mathcal{A}}^{\mathcal{M}}\left(D_{\mathcal{M}} E_{\mathcal{C})}{ }^{\mathcal{N}}\right) E_{\mathcal{N}}^{-1 \mathcal{E}} \\
& +\frac{1}{2} \eta^{\mathcal{E} \mathcal{D}} E_{\mathcal{D}}{ }^{\mathcal{M}}\left(D_{\mathcal{M}} E_{\left[\left.\mathcal{A}\right|^{\mathcal{N}}\right.}\right) E_{\mathcal{N}}^{-1 \mathcal{F}} \eta_{\mathcal{F} \mid \mathcal{C})}+E_{\mathcal{A}}{ }^{\mathcal{M}} E_{\mathcal{C}}{ }^{\mathcal{N}} E_{\mathcal{P}}^{-1 \mathcal{E}} f_{\mathcal{M N}}{ }^{\mathcal{P}}
\end{aligned}
$$


where $[\mathcal{A}|| \mathcal{C})$ indicates graded antisymmetrization in only those indices. By $\mathcal{D}_{\mathcal{M}}$ in (3.11) and in the whole text we mean the group covariant derivatives of the (non-affine) part of algebra (3.2): $\left[\mathcal{D}_{\mathcal{M}}, \mathcal{D}_{\mathcal{N}}\right\}=-i f_{\mathcal{M} \mathcal{N}^{\mathcal{E}}} \mathcal{D}_{\mathcal{E}}$.

Note that the (super)Jacobi identities imply the total graded antisymmetry of the torsion, just as for the structure constants. Torsion (3.11) can be identified with that of "ordinary" curved-space covariant derivatives by use of the strong constraint, as explained in $[1-4]$.

We can set the coefficient of the Schwinger term to be the metric $\eta$; the vielbein is forced to obey the orthogonality constraints:

$$
E_{\mathcal{A}}{ }^{\mathcal{M}} \eta_{\mathcal{M N}} E_{\mathcal{C}}^{\mathcal{N}} \equiv \eta_{\mathcal{A C}}
$$

This choice does not affect the physics, and simplifies many of the expressions. For example, it implies the total graded antisymmetry of the torsion, when the upper index is implicitly lowered with $\eta$ :

$$
T_{\mathcal{A B C}}=\frac{1}{2} E_{[\mathcal{A} \mid} \mathcal{M}^{\left.\left(D_{\mathcal{M}} E_{\mid \mathcal{B}}{ }^{\mathcal{N}}\right) E_{\mathcal{C}}\right) \mathcal{N}}+E_{\mathcal{A}}{ }^{\mathcal{M}} E_{\mathcal{B}}{ }^{\mathcal{N}} E_{\mathcal{C}}{ }^{\mathcal{P}} f_{\mathcal{M N} \mathcal{N}}
$$

where we have used $E_{\mathcal{M}}^{-1}{ }^{\mathcal{A}}=\eta^{\mathcal{A}}{ }^{\mathcal{B}} \eta_{\mathcal{M} \mathcal{N}} E_{\mathcal{B}^{\mathcal{N}}}$. (Also note that in the first term the graded antisymmetrization can be written as a cyclic sum without the $1 / 2$, since it is already graded antisymmetric in the last two indices.) Thus, because of orthogonality, the vielbein is like (the exponential of) a super 2-form, while the torsion is a super 3-form; similarly, the Bianchi identities are a super 4-form.

The (super)orthogonality constraint (3.12) can be fully solved for the general structure of the vielbein $E_{\mathcal{A}} \mathcal{M}$. However, we are interested just in the linear level. Thus we get the (super)orthogonality constraint for the linearised part of the vielbein $E^{(1)} \mathcal{A}^{\mathcal{M}}$ :

$$
\begin{aligned}
E_{\mathcal{A}} \mathcal{M} & =\delta_{\mathcal{A}} \mathcal{M}+E^{(1)} \mathcal{A}^{\mathcal{M}}+\mathcal{O}\left(E^{(2)}\right) \Rightarrow \text { using (3.12) } \\
E^{(1)}(\mathcal{A B}] & =0
\end{aligned}
$$

We would also need the linear level version of the equation (3.13):

$$
\begin{aligned}
T_{\mathcal{A B C}} & =f_{\mathcal{A B C}}+T^{(1)} \mathcal{A B C}+\mathcal{O}\left(E^{(2)}\right) \\
\text { where } T^{(1)}{ }_{\mathcal{A B C}} & :=\frac{1}{2} D_{[\mathcal{A}} E^{(1)}{ }_{\mathcal{B C})}+\frac{1}{2} E^{(1)}\left[\mathcal{A}^{\mathcal{M}} f_{\mathcal{M} \mid \mathcal{B C})}\right.
\end{aligned}
$$

\subsection{Further constraints and gauge fixing}

Following the discussion in subsection 4.2 in the paper [1], we get the coset constraint on the torsion piece $T_{\mathcal{S}} \mathcal{A}^{\mathcal{B}}=f_{\mathcal{S}} \mathcal{A}^{\mathcal{B}}$ (where we used the $\mathcal{S}$ index as the schematic index (3.4) and $\mathcal{A}, \mathcal{B}$ are general indices). At the linear level the previous condition becomes: $T^{(1)} \mathcal{S} \mathcal{A B}=0$. From this one gets the condition for the linear vielbein: $E^{(1)} \mathcal{S}^{\mathcal{M}}=0+\mathcal{O}\left(E^{(2)}\right)$.

We would like to gauge fix some of the remaining gauge freedom. Note that the coset constraints discussed above sets the gauge parameter (defined below) $\lambda_{\mathcal{S}}=0$. From specific gauge fixing we get further conditions on the linear vielbein $E^{(1)}$. The gauge transformations are given as (see also [2-4]):

$$
\delta_{\Lambda} \Pi_{\mathcal{A}}=\left[-i \Lambda, \Pi_{\mathcal{A}}\right\}
$$




$$
\text { where } \Lambda:=\int d 1 \lambda^{\mathcal{M}}(X) D_{\mathcal{M}}
$$

We are working in the basis where the covariant derivatives satisfy: $\left[D_{\mathcal{M}}, D_{\mathcal{N}}\right\}=$ $i f_{\mathcal{M N}} \mathcal{N}^{\mathcal{P}} D_{\mathcal{P}}$. Thus the (linear) gauge transformation of (linear) vielbein are:

$$
\delta_{\Lambda} E^{(1)} \mathcal{A B}=-\frac{i}{2} D_{[\mathcal{A}} \lambda_{\mathcal{B})}+f_{\mathcal{A B}}{ }^{\mathcal{C}} \lambda_{\mathcal{C}}
$$

Now, we can pick the following gauge:

$$
\begin{aligned}
\gamma_{\mathbf{a}}{ }^{\alpha \beta} E^{(1)}{ }_{\alpha \beta}=0 & \Rightarrow \lambda_{\mathbf{a}} \propto \gamma_{\mathbf{a}}^{\alpha \beta} D_{\alpha} \lambda_{\beta} \\
\gamma^{\mathbf{a} \alpha \beta} E^{(1)}{ }_{\alpha \mathbf{a}}=0 & \Rightarrow \lambda^{\alpha} \propto \gamma^{\mathbf{a} \alpha \beta} D_{[\mathbf{a}} \lambda_{\beta]} \\
E^{(1)}{ }_{\mathbf{a} \mathbf{b}}=0 & \Rightarrow \lambda_{\mathbf{a} \mathbf{b}} \propto D_{[\mathbf{a}} \lambda_{\mathbf{b}]} \\
& \longrightarrow \text { Same for Left } \rightarrow \text { Right }
\end{aligned}
$$

We can see that by (3.20), (3.21), (3.22) we automatically have expressions for gauge parameters $\lambda_{P}, \lambda_{\Omega}, \lambda_{\Sigma}$ as derivatives of another gauge parameter $\lambda_{D}$. It is unlike the usual $\mathcal{N}=1$ supergravity where we need first to solve the chirality condition to relate derivatives of $\Lambda$ with $K$, see section X.A.1 in [20], also section 5.3 in [17]. Moreover, (3.20)(3.22) give the constraints on $E^{(1)}$ and solving those we will get:

$$
E_{D D}^{(1)}=E_{\alpha \beta}^{(1)}=0, \quad E^{(1)} P P=E^{(1)}{ }_{\mathbf{a b}}=0, \quad E^{(1) \alpha \beta}{ }_{\beta}=0\left(\text { part of } E^{(1) P}{ }_{D}\right)
$$

Later (by dimension $-\frac{1}{2}$ constraints) one can see that $E^{(1)} P D=0$. We thus need to set up the dimensional constraints. The table 1 summarise the torsion dimensions.

We put the torsions of negative (engineering) dimensions to 0 (as always in QFT, see the red coloured torsions in table 1 ). We also put the (unfixed) torsions of zero dimension to 0 (see the blue torsions in the previous table), see [1]. We will also put the dimension $\frac{1}{2}$ (unfixed) torsions to 0 (the green torsions in the table). Doing that we produce just algebraic constraints on veilbeins.

The nontrivial dimensional constraints are:

$$
T_{D}{ }^{\Omega}=0, T_{D D}{ }^{P}=f_{D D}{ }^{P}, T_{D}{ }^{D}=0, T_{P} P^{\Omega}=0
$$

\subsection{Dimensional constraints: solution}

The solution to the previous nontrivial dimensional constraints can be given in full generality; however in this paper we are interested just in the linearised case.

Tables 2 and 3 summarise the linearised solutions of those four constraints (3.24). Notice that we have also the possibility of mixed left/right indices. (Notice also the "." in the tables 2 and 3 means symbolic contraction. Do not be confused with "." defined in $(3.7))$.

From table 2 we can see that we have one linear relation: $E^{(1)} \Sigma D \propto E^{(1)} \Omega P$. From table 3 we have linear relations: $\left\{E_{P \tilde{D}}^{(1)}, E_{P \tilde{P}}^{(1)}, E_{\Omega \tilde{D}}^{(1)}, E_{\Omega \tilde{P}}^{(1)}, E_{\Sigma \tilde{D}}^{(1)}\right\} \propto E^{(1)}{ }_{D} \tilde{D}^{\text {. }}$ Again, we have automatically obtained the expressions for the vielbeins as derivatives of the $E^{(1)} D \tilde{D}$ vielbein (prepotential). It is unlike $\mathcal{N}=1$ supergravity where the prepotential comes as the solution of the bisection condition (or chirality condition in covariant approach), see section X.A.1 in [20] and section 5.2.a and 5.3 in [17]. 


\begin{tabular}{|cc|}
\hline Torsion & Dim. \\
\hline$T_{S S}{ }^{\Sigma}$ & -2 \\
\hline$T_{S S}{ }^{P}$ & $-\frac{3}{2}$ \\
\hline$T_{S S}{ }^{P}$ & -1 \\
\hline$T_{S D^{\Omega}}$ & -1 \\
\hline$T_{S S^{D}}$ & $-\frac{1}{2}$ \\
\hline$T_{S P}{ }^{\Omega}$ & $-\frac{1}{2}$ \\
\hline$T_{D}{ }^{\Omega}$ & $-\frac{1}{2}$ \\
\hline$T_{S S}{ }^{S}$ & 0 \\
\hline$T_{S D}{ }^{D}$ & 0 \\
\hline$T_{S P}{ }^{P}$ & 0 \\
\hline$T_{D D}{ }^{P}$ & 0 \\
\hline
\end{tabular}

\begin{tabular}{|c|c|}
\hline Torsion & Dim. \\
\hline$T_{S D}{ }^{S}$ & $\frac{1}{2}$ \\
\hline$T_{S P}{ }^{D}$ & $\frac{1}{2}$ \\
\hline$T_{D D}{ }^{D}$ & $\frac{1}{2}$ \\
\hline$T_{P} P^{\Omega}$ & $\frac{1}{2}$ \\
\hline$T_{S P}^{S}$ & 1 \\
\hline$T_{S} \Omega^{D}$ & 1 \\
\hline$T_{D P}{ }^{D}$ & 1 \\
\hline$T_{D D}{ }^{S}$ & 1 \\
\hline$T_{P P}^{P}$ & 1 \\
\hline$T_{S \Omega} S$ & $\frac{3}{2}$ \\
\hline$T_{D P} S$ & $\frac{3}{2}$ \\
\hline$T_{D \Omega}{ }^{D}$ & $\frac{3}{2}$ \\
\hline$T_{P P}{ }^{D}$ & $\frac{3}{2}$ \\
\hline
\end{tabular}

\begin{tabular}{|cc|}
\hline Torsion & Dim. \\
\hline$T_{S \Sigma}{ }^{S}$ & 2 \\
\hline$T_{D \Omega}{ }^{S}$ & 2 \\
\hline$T_{P P}{ }^{S}$ & 2 \\
\hline$T_{P \Omega}{ }^{D}$ & 2 \\
\hline$T_{D \Sigma} S$ & $\frac{5}{2}$ \\
\hline$T_{P \Omega} S$ & $\frac{5}{2}$ \\
\hline$T_{\Omega \Omega}{ }^{D}$ & $\frac{5}{2}$ \\
\hline$T_{P \Sigma} S$ & 3 \\
\hline$T_{\Omega \Omega}{ }^{S}$ & 3 \\
\hline$T_{\Omega \Sigma} S$ & $\frac{7}{2}$ \\
\hline$T_{\Sigma \Sigma} S$ & 4 \\
\hline
\end{tabular}

Table 1. Torsion dimensions.

\begin{tabular}{|cllc|}
\hline$T_{D D^{\Omega}}=0$ and $\gamma^{\mathbf{a} \alpha \beta} E^{(1)}{ }_{\alpha \mathbf{a}}=0$ & $\Rightarrow$ & $E^{(1)}{ }_{P D}=0$ \\
\hline$T_{D D^{P}}=f_{D D^{P}}$ & $\Rightarrow$ & $E^{(1)}{ }_{D \Omega}=0$ \\
\hline$T_{P} P^{\Omega}=0$ or $T_{D} D^{D}=0$ & $\Rightarrow$ & $E^{(1)}{ }_{\Sigma D}=E^{(1) \mathbf{a b}}{ }_{\alpha}=-2 \gamma^{[\mathbf{a}}{ }_{\alpha \rho} E^{(1) \rho \mid \mathbf{b}]}$ \\
& & & $\equiv \gamma \cdot E^{(1)}{ }_{\Omega}^{P}$ \\
\hline
\end{tabular}

Table 2. Unmixed constraints.

\begin{tabular}{|c|c|}
\hline$T_{D} D^{\tilde{\Omega}}=0$ & 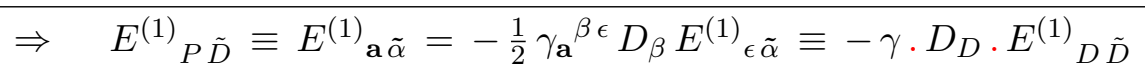 \\
\hline$T_{D D}{ }^{\tilde{P}}=0$ & $E_{P \tilde{P}}^{(1)} \equiv E_{\mathbf{a} \tilde{\mathbf{b}}}^{(1)}=-\frac{1}{2} \gamma_{\mathbf{a}}^{\beta \epsilon} D_{\beta} E_{\epsilon \tilde{\mathbf{b}}}^{(1)} \equiv-\gamma \cdot D_{D} \cdot E^{(1)}{ }_{D \tilde{P}}$ \\
\hline$T_{D \tilde{D}}^{P}=0$ & $\Rightarrow \quad E_{\Omega \tilde{D}}^{(1)}{ } \equiv E_{\tilde{\beta}}^{(1) \alpha_{\tilde{\beta}}}=-\frac{1}{6} \gamma^{\mathbf{a} \epsilon \alpha} D_{[\epsilon} E_{\mathbf{a}] \tilde{\beta}}^{(1)} \equiv-\gamma \cdot D_{[D} \cdot E_{P] \tilde{D}}^{(1)}$ \\
\hline$T_{P \tilde{P}^{\Omega}}=0$ & $\left.\Rightarrow \quad E_{\Omega \tilde{P}}^{(1)} \equiv E^{(1) \alpha} \tilde{\mathbf{a}}=-\frac{1}{6} \gamma^{\mathbf{b} \epsilon \alpha} D_{[\epsilon} E^{(1)} \mathbf{b}\right] \tilde{\mathbf{a}} \equiv-\gamma \cdot D_{[D} \cdot E_{P] \tilde{P}}^{(1)}$ \\
\hline$T_{P P} \tilde{\Omega}=0$ & $\left.E^{(1) \mathbf{a b}} \tilde{\alpha}=\eta^{\mathbf{a} \mathbf{c}} \eta^{\mathbf{b} \mathbf{d}} D_{[\mathbf{c}} E^{(1)} \mathbf{d}\right] \tilde{\alpha} \equiv \eta$ \\
\hline
\end{tabular}

Table 3. Mixed constraints. 


\subsection{Dimension 1 unmixed constraints}

To proceed we need to find the constraints for the dimension 1 torsions. We can see that putting those to zero in general introduces differential constraints, which we do not want (except for the strong constraint and later the equation of motion). However, there is a way how to fix dimension 1 torsions without producing differential constraints. We will use the following set of unmixed constraints (again we have two cases for the torsion index structure, mixed and unmixed):

$$
\begin{aligned}
& T^{(1)}{ }_{P P P} \equiv T^{(1)} \mathbf{a b} \mathbf{c}=\vartheta \varepsilon_{\mathbf{a} \mathbf{b} \mathbf{c}} B \\
& T^{(1)} D D \Sigma \\
& T^{(1)} T^{(1)}{ }_{\alpha \beta} \mathbf{a b}=\xi \gamma^{\mathbf{a b}}{ }_{\alpha \beta} B \\
& T^{(1)}{ }_{\mathbf{a} \alpha}{ }^{\beta}=\zeta \gamma_{\mathbf{a}}{ }_{\alpha} B
\end{aligned}
$$

where the new object $B$ is determined from (3.25). Using the linearised Bianchi identity we get:

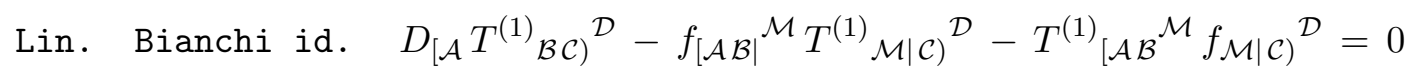

For dim. 1: $\quad \frac{1}{2} T^{(1)}{ }_{\alpha \beta}{ }^{\mathbf{a b}}-\gamma^{[\mathbf{a}}{ }_{(\alpha \mid \epsilon} T^{(1)}{ }_{\beta)}^{\epsilon \mid \mathbf{b}]}+\gamma_{\alpha \beta}^{\mathbf{c}} T^{(1)} \mathbf{c}^{\mathbf{a b}}=0$

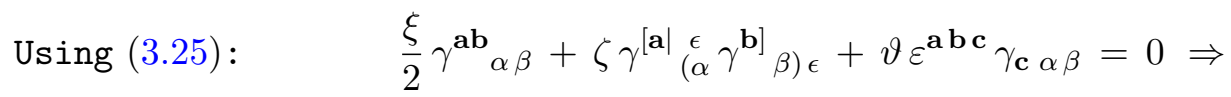

$$
\xi=-8 \zeta-2 \vartheta
$$

Equation (3.17) gives explicit relations for the $T^{(1)}$ 's from (3.25):

$$
\begin{aligned}
& T_{\mathbf{a} \mathbf{b} \mathbf{c}}^{(1)}=-\frac{1}{2} \eta_{[\mathbf{a} \mid \mathbf{d}} \eta_{\mathbf{b} \mid \mathbf{e}} E^{(1) \mathbf{d e}]} \equiv-\eta \eta \cdot E^{(1)}{ }_{\Sigma P} \\
& T^{(1)}{ }_{\alpha \beta}{ }^{\mathbf{a b}}=D_{(\alpha} E^{(1)}{ }_{\beta)}{ }^{\mathbf{a b}}+2 \gamma_{\alpha \beta}^{\mathbf{c}} E^{(1) \mathbf{a b}}{ }_{\mathbf{c}} \equiv D \cdot E^{(1)} D \Sigma+\gamma \cdot E^{(1)} \Sigma P \\
& T^{(1)}{ }_{\mathbf{a} \alpha}^{\beta}=D_{\alpha} E_{\mathbf{a}}^{(1) \beta}+2 \gamma_{\mathbf{a} \alpha \epsilon} E^{(1) \epsilon \beta}+\frac{1}{4} \varepsilon_{\mathbf{c d e} \mathbf{e}} \gamma_{\alpha}^{\mathbf{e} \beta} E^{(1) \mathbf{c d}} \mathbf{a}_{\mathbf{a}} \\
& \equiv D \cdot E^{(1)} \Omega P+\gamma \cdot E^{(1)} \Omega \Omega+\varepsilon \gamma \cdot E^{(1)} \Sigma P
\end{aligned}
$$

From (3.27) and first relation of (3.25) we get:

$$
2 \vartheta B=-\varepsilon_{\mathbf{h d ~} \mathbf{e}} \eta^{\mathbf{h} \mathbf{c}} E^{(1) \mathbf{d e}} \mathbf{c}
$$

From (3.29) and requiring that we want just algebraic constraints we get the second equation for (3.25) fixing constants:

$$
0=-3 \zeta-\frac{1}{8}(2 \vartheta+3 \xi)-\frac{1}{2} \vartheta
$$

Substituing result (3.26) we have the solution for any $\vartheta$ and $\zeta$ except when $\vartheta=-6 \zeta$. That condition would produce a differential constraint on $E^{(1)} \Omega P$ (see eq. (3.32)). From (3.29) and the third of (3.25) we will get fixing of $E^{(1)} \Omega \Omega$. From (3.28) and the second of (3.25) we will get fixing of $E^{(1)} P \Sigma$. The net result of dimension 1 unmixed algebraic constraints (3.25) 
is that everything can be expressed in terms of $E^{(1)} D \Sigma$ and so (see table 2) by $E^{(1)} P \Omega$ (and two constants $\vartheta, \zeta$ s.t. $\vartheta \neq-6 \zeta)$ :

$$
\begin{aligned}
B & =-\frac{1}{\vartheta+6 \zeta} \gamma_{\beta}^{\mathbf{a} \alpha} D_{\alpha} E_{\mathbf{a}}^{(1) \beta} \\
& \equiv-\gamma D_{D} \cdot E^{(1)}{ }_{\Omega P} \\
E^{(1)} \Omega \Omega=E^{(1) \alpha \beta} & =\frac{1}{12} \gamma^{\mathbf{a}(\alpha \mid \epsilon} D_{\epsilon} E^{(1) \beta)} \mathbf{a}+\frac{1}{12} \gamma_{\mathbf{a}}^{\alpha \beta} E^{(1) \mathbf{a b}}{ }_{\mathbf{b}} \\
& \equiv \gamma D_{D} \cdot E^{(1)} \Omega P+\gamma \cdot E_{\Sigma P}^{(1)} \\
E_{P \Sigma}^{(1)}=E^{(1)} \mathbf{c}^{\mathbf{a b}} & =-\frac{1}{2} \gamma_{\mathbf{c}}^{\alpha \beta} D_{\alpha} E_{\beta}^{(1)}{ }^{\mathbf{a b}}+(\vartheta+4 \zeta) \eta_{\mathbf{c}} \mathbf{e} \varepsilon^{\mathbf{e a b}} B \\
& \equiv-\gamma D_{D} \cdot E^{(1)}{ }_{D \Sigma}+\eta \varepsilon \cdot B
\end{aligned}
$$

\subsection{Dimension 1 mixed constraints}

Some of the mixed dimension 1 torsions are determined in terms of $E^{(1)}{ }_{\alpha \tilde{\beta}} \equiv E^{(1)}{ }_{D} \tilde{D}$ and $E^{(1)} P \Omega$ already. Using the previous results (tables 2,3 and results of the previous section) we can see that mixed dimension 1 torsions $T^{(1)}{ }_{\mathbf{a} \tilde{\alpha}^{\rho}} \equiv T_{P \tilde{D} \Omega}^{(1)}$ and $T^{(1)} \tilde{\alpha} \beta{ }^{\text {ab }} \equiv T^{(1)} \tilde{D} D \Sigma$ are fully determined, see (3.35). The mixed determined and undetermined torsions are summarised below:

$$
\begin{aligned}
& \left.\begin{array}{l}
\left.T_{P \tilde{D} \Omega}^{(1)} \equiv T^{(1)}{ }_{\mathbf{a} \tilde{\alpha}^{\beta}}=D_{\mathbf{a}} E^{(1)} \tilde{\alpha}^{\beta}+D^{(\beta} E^{(1)}{ }_{\tilde{\alpha}) \mathbf{a}} \equiv D_{P} E^{(1)}{ }_{\tilde{D} \Omega}+D_{(\Omega} E^{(1)} \tilde{D}\right) P \\
T_{\tilde{D} D \Sigma}^{(1)} \equiv T_{\tilde{\alpha} \beta}^{(1)}{ }^{\mathbf{a b}}=D_{(\tilde{\alpha}} E^{(1)}{ }_{\beta)}^{\mathbf{a b}}+D^{\mathbf{a b}} E_{\tilde{\alpha} \beta}^{(1)} \equiv D_{(\tilde{D}} E^{(1)}{ }_{D) \Sigma}+D_{\Sigma} E^{(1)}{ }_{\tilde{D} D}
\end{array}\right\} \\
& \left.T_{\tilde{P} P P}^{(1)} \equiv T^{(1)} \tilde{\mathbf{a}}_{\mathbf{b} \mathbf{c}}=D_{[\mathbf{b}} E^{(1)} \mathbf{c}\right] \tilde{\mathbf{a}}-\eta_{\mathbf{b} \mathbf{d}} \eta_{\mathbf{c} \mathbf{e}} E^{(1) \mathbf{d e}_{\tilde{\mathbf{a}}}} \equiv D_{[P} E_{P] \tilde{P}}^{(1)}-\eta \eta \cdot E_{\Sigma \tilde{P}}^{(1)}
\end{aligned}
$$

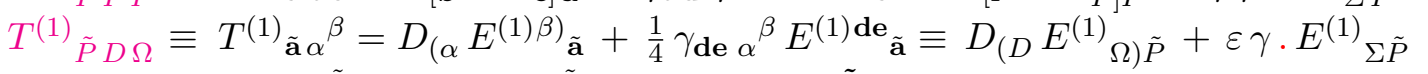

$$
\begin{aligned}
& T_{P D \tilde{\Omega}}^{(1)} \equiv T^{(1)} \mathbf{a} \alpha^{\tilde{\beta}}=D_{[\mathbf{a}} E^{(1)}{ }_{\alpha]}^{\tilde{\beta}}+2 \gamma_{\mathbf{a} \alpha \epsilon} E^{(1) \epsilon \tilde{\beta}} \equiv D_{[P} E_{D] \tilde{\Omega}}^{(1)}+\gamma \cdot E^{(1)}{ }_{\Omega \tilde{\Omega}}
\end{aligned}
$$

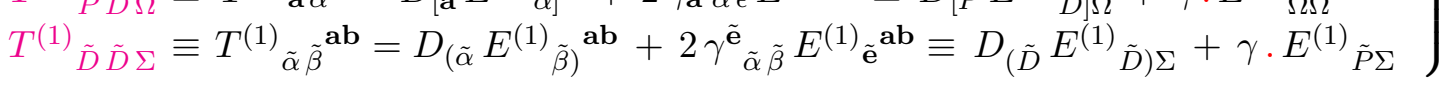

From (3.36) it is evident that by putting $T^{(1)}{ }_{\tilde{P} P P}=0$ we can determine $E^{(1)}{ }_{\Sigma} \tilde{P}$ in terms of $E^{(1)} P \tilde{P}$ and so $E^{(1)} D \tilde{D}$. Equivalently we can obtain that by fixing either of $T^{(1)} \tilde{P} D \Omega$ or $T^{(1)}{ }_{D} D \tilde{\Sigma}$. By putting $T^{(1)} P D \tilde{\Omega}=0$ we can determine $E^{(1)}{ }_{\Omega} \tilde{\Omega}$ in terms of $E^{(1)}{ }_{D} \tilde{\Omega}$ and $E_{P \tilde{\Omega}}^{(1)}$ and so again in $E^{(1)} D \tilde{D}$. The dimension 1 mixed constraints give:

$$
\begin{aligned}
& E_{\Sigma \tilde{P}}^{(1)} \equiv E^{(1) \mathbf{b} \mathbf{c}_{\tilde{\mathbf{a}}}}=\eta^{\mathbf{b} \mathbf{d}} \eta^{\mathbf{c} \mathbf{e}} D_{[\mathbf{d}} E^{(1)} \mathbf{e} \tilde{\mathbf{a}} \equiv \eta \eta \cdot D_{[P} E^{(1)}{ }_{P] \tilde{P}} \\
& E_{\Omega \tilde{\Omega}}^{(1)} \equiv E^{(1) \alpha \tilde{\beta}}=\frac{1}{6} \gamma^{\mathbf{a} \alpha \epsilon} D_{[\mathbf{a}} E_{\epsilon]}^{(1)} \equiv \gamma \cdot D_{[P} E_{D] \tilde{\Omega}}^{(1)}
\end{aligned}
$$

The dimension 1 constraints can be viewed also from another perspective. For that we need to borrow the expression for the Cartan-Killing metric $K_{\mathcal{A B}}$ that is discussed in section 4.1. The expression for the linearised Cartan-Killing metric is:

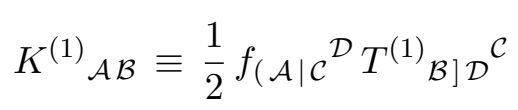


Taking (3.39) for $\mathcal{A}, \mathcal{B} \in\{\alpha, \tilde{\beta}\}$ we get:

$$
K_{\alpha \beta}^{(1)} \propto \varepsilon_{\alpha \beta} B, K_{\tilde{\alpha} \tilde{\beta}}^{(1)} \propto \varepsilon_{\tilde{\alpha} \tilde{\beta}} \tilde{B}, K_{\alpha \tilde{\beta}}^{(1)}
$$

Then using exercise XA2.6 in [20] we can write the dimension 1 constraints as:

$$
\begin{array}{rlrl}
T_{\mathbf{a b} \mathbf{c}}^{(1)} & \propto \varepsilon_{\mathbf{a b} \mathbf{b}} \varepsilon^{\alpha \beta} K_{\beta \alpha}^{(1)}, & T_{\alpha \beta}^{(1)}{ }_{\alpha \beta}^{\mathbf{a b}} \propto \gamma_{\alpha \beta}^{\mathbf{a b}} \varepsilon^{\epsilon \sigma} K_{\sigma \epsilon}^{(1)} \\
T^{(1)} \mathbf{a}^{\beta}{ }^{\beta} \propto \gamma_{\mathbf{a} \alpha}^{\beta} \varepsilon^{\epsilon \sigma} K_{\sigma \epsilon}^{(1)}, & T^{(1)} \mathbf{a} \tilde{\alpha}^{\beta} & \propto \gamma_{\mathbf{a}}{ }^{\beta \epsilon} K^{(1)}{ }_{\epsilon \tilde{\alpha}} \\
T_{\tilde{\alpha} \beta}^{(1)} & \propto \gamma^{\mathbf{a b}}{ }_{\beta}^{\epsilon} K^{(1)}{ }_{\epsilon \tilde{\alpha}}, & T^{(1)} \mathbf{a} \alpha^{\tilde{\beta}} & \propto \gamma_{\mathbf{a} \alpha \epsilon} K^{(1) \epsilon \tilde{\beta}}
\end{array}
$$

Remaining dimension 1 torsions have to be 0 since we do not have an appropriate nonzero Cartan-Killing metric. We also put the second torsion of (3.43) to 0, since that does not produce any differential constraints and fixes $E^{(1)}{ }_{\Omega \tilde{\Omega}}$, see (3.38). Moreover in the spirit of exercise XA2.6 in [20], we can identify $\left(K^{(1)}{ }_{\alpha \tilde{\beta}}, B, \tilde{B}\right)$ with a $\mathrm{SO}(3,3)$ vector $G^{\alpha \beta}=\left(G^{a}, B, \bar{B}\right)$ in $\operatorname{SL}(4)$ notation (from $\mathcal{N}=1$ supergravity).

\section{$3.7 \tilde{T}=0$}

In the previous subsections we discovered that all the vielbeins (mixed and unmixed, except for $E^{(1)} \Omega \Sigma$ and $E^{(1)} \Sigma \Sigma$ ) can be determined in terms of $E^{(1)} P \Omega$ and $E^{(1)} D \tilde{D}$. We need further constraints to relate those two undetermined vielbeins. We are following article [24]. There a new torsion was introduced. It came from the requirement of partial integration also in the presence of the new integration measure $\phi^{2}$ (dilaton). Following [2-4] the new torsion is:

$$
\tilde{T}_{\mathcal{A}}:=\phi^{2} \overleftarrow{\nabla}_{\mathcal{A}} \phi^{-2}
$$

where $\nabla_{\mathcal{A}}=E_{\mathcal{A}}{ }^{\mathcal{M}} D_{\mathcal{M}}$. The torsion (3.44) should vanish, so we get the $\tilde{T}$ torsion constraint $\tilde{T}_{\mathcal{A}}=0$. We are interested just in the first order part of $\tilde{T}_{\mathcal{A}}$ :

$$
\begin{gathered}
\tilde{T}_{\mathcal{A}}=0+\tilde{T}_{\mathcal{A}}^{(1)}+\mathcal{O}\left(E^{(2)}\right) \Rightarrow \tilde{T}_{\mathcal{A}}^{(1)}=D^{\mathcal{B}} E^{(1)} \mathcal{B \mathcal { A }}+2 D_{\mathcal{A}} \phi^{(1)} \\
\text { where } \quad \phi=1+\phi^{(1)}+\mathcal{O}\left(\phi^{(2)}\right)
\end{gathered}
$$

The relation $\tilde{T}^{(1)}{ }_{S}=0$ gives $D_{S} \phi^{(1)}=0$. Using $\tilde{T}^{(1)}{ }_{D}=0$ we get the relation:

$$
\begin{aligned}
\frac{1}{4} \varepsilon_{\mathbf{a b} \mathbf{c}} \gamma_{\alpha}^{\mathbf{c} \beta} E_{\beta}^{(1) \mathbf{a b}}=2 \gamma_{\mathbf{a} \alpha \beta} E^{(1) \beta \mathbf{a}}= & D_{\tilde{\beta}}^{\tilde{\beta}} E_{\tilde{\beta} \alpha}^{(1)}+D_{\tilde{\mathbf{a}}}^{E^{(1)} \tilde{\mathbf{a}} \alpha} \\
& -D_{\tilde{\beta}} E_{\alpha}^{(1) \tilde{\beta}}-2 D_{\alpha} \phi^{(1)} \\
\gamma \cdot E_{\Omega P}^{(1)}= & D_{\tilde{\Omega}} E_{\tilde{D} D}^{(1)}+D_{\tilde{P}} E_{\tilde{P} D}^{(1)}-D_{\tilde{D}} E_{\tilde{\Omega} D}^{(3)} \\
& -D_{D} \phi^{(1)}
\end{aligned}
$$

where we used the results of table 2. Using table 3 for $E^{(1)} \tilde{a} \alpha$ and $E^{(1)} \tilde{\beta}_{\alpha}$ we have the relation between $E^{(1)} P \Omega$ and $E^{(1)} D \tilde{D}$ and the linearised dilaton $\phi^{(1)}$ :

$$
\begin{aligned}
& \gamma \cdot E_{\Omega P}^{(1)} \equiv 2 \gamma_{\alpha \beta}^{\mathbf{a}} E^{(1) \beta} \mathbf{a}=-\frac{1}{3} \gamma^{\tilde{\mathbf{a}} \tilde{\beta} \tilde{\epsilon}}\left[D_{\tilde{\mathbf{a}}}, D_{\tilde{\beta}}\right] E^{(1)} \tilde{\epsilon} \alpha+\frac{1}{2} \gamma^{\tilde{\mathbf{a}} \tilde{\beta} \tilde{\epsilon}} D_{\tilde{\mathbf{a}}} D_{\tilde{\beta}} E^{(1)} \tilde{\epsilon} \alpha \\
& -2 D_{\alpha} \phi^{(1)} \\
& \equiv-\gamma \cdot\left[D_{\tilde{P}}, D_{\tilde{D}}\right] E^{(1)}{ }_{\tilde{D} D}+\gamma \cdot D_{\tilde{P}} D_{\tilde{D}} E^{(1)}{ }_{\tilde{D} D}-D_{D} \phi^{(1)}
\end{aligned}
$$


We notice that result (3.47) is exactly the right combination in order to express $B$ from (3.32) in terms of $E^{(1)} \tilde{D} D$ and $\phi^{(1)}$. This will be used in following sections:

$$
\begin{aligned}
B & =-\frac{1}{\vartheta+6 \zeta} \varepsilon^{\nu \alpha} D_{\nu}\left[\gamma^{\tilde{\mathbf{a}} \tilde{\beta} \tilde{\epsilon}}\left(-\frac{1}{6}\left[D_{\tilde{\mathbf{a}}}, D_{\tilde{\beta}}\right]+\frac{1}{4} D_{\tilde{\mathbf{a}}} D_{\tilde{\beta}}\right) E_{\tilde{\epsilon} \alpha}^{(1)}-D_{\alpha} \phi^{(1)}\right] \\
& \equiv \varepsilon \cdot D_{D}\left[\gamma \cdot\left(\left[D_{\tilde{P}}, D_{\tilde{D}}\right]-D_{\tilde{P}} D_{\tilde{D}}\right) E^{(1)} \tilde{D} D-D_{D} \phi^{(1)}\right]
\end{aligned}
$$

Using the relation $\tilde{T}^{(1)} P=0$ and similar steps we get:

$$
\begin{aligned}
-2 \varepsilon_{\mathbf{a b} \mathbf{c}} \gamma_{\epsilon}^{\mathbf{b} \alpha} D_{\alpha} E^{(1) \epsilon \mathbf{c}} & +2 D_{\alpha} E_{\mathbf{a}}^{(1) \alpha}-4 D_{\mathbf{a}} \phi^{(1)} \\
= & -\frac{1}{3} \gamma^{\tilde{\mathbf{a}} \tilde{\beta} \tilde{\epsilon}}\left[D_{\tilde{\mathbf{a}}}, D_{\tilde{\beta}}\right] \gamma_{\mathbf{a}}^{\sigma \alpha} D_{\sigma} E^{(1)} \tilde{\epsilon} \alpha \\
& +\frac{1}{2} \gamma^{\tilde{\mathbf{a}} \tilde{\beta} \tilde{\epsilon}} D_{\tilde{\mathbf{a}}} D_{\tilde{\beta}} \gamma_{\mathbf{a}}^{\sigma \alpha} D_{\sigma} E^{(1)} \tilde{\epsilon} \alpha \\
(\operatorname{using}(3.47))= & \gamma_{\mathbf{a}}^{\sigma \alpha} D_{\sigma}\left(-2 \gamma_{\alpha \beta}^{\mathbf{b}} E^{(1) \beta_{\mathbf{b}}}\right) \\
-2 \varepsilon_{\mathbf{a} \mathbf{b} \mathbf{c}} \gamma_{\epsilon}^{\mathbf{b} \alpha} D_{\alpha} E^{(1) \epsilon \mathbf{c}} & +2 D_{\alpha} E_{\mathbf{a}}^{(1) \alpha}-4 D_{\mathbf{a}} \phi^{(1)} \\
= & -2 \varepsilon_{\mathbf{a} \mathbf{b} \mathbf{c}} \gamma_{\epsilon}^{\mathbf{b} \alpha} D_{\alpha} E^{(1) \epsilon \mathbf{c}}+2 D_{\alpha} E^{(1) \alpha} \mathbf{a} \\
& -4 D_{\mathbf{a}} \phi^{(1)}
\end{aligned}
$$

So, from the relation $\tilde{T}^{(1)}{ }_{P}=0$ we will get no new constraints.

From relations $\tilde{T}_{\Omega}^{(1)}=0$ and $\tilde{T}_{\Sigma}^{(1)}{ }_{\Sigma}=0$ we will get some constraints on unfixed (and unused) vielbeins $E^{(1)} \Sigma \Omega$ and $E^{(1)} \Sigma \Sigma$.

\section{Cartan-Killing metric and field equations}

\subsection{Cartan-Killing metric}

Having the Lie algebra $\mathcal{G}$, one can define a symmetric bilinear form:

$$
\begin{aligned}
& K(X, Y):=\frac{1}{x_{\lambda}} \operatorname{Tr}\left(\operatorname{ad}_{X} \operatorname{ad}_{Y}\right) \equiv \frac{1}{x_{\lambda}}\left\langle E^{i}\left|\operatorname{ad}_{X} \operatorname{ad}_{Y}\right| E_{i}\right\rangle \\
& \text { where } \quad X, Y \in \mathcal{G} \text { and } x_{\lambda} \equiv \text { Dynkin index } \\
& \text { and } E_{i}, E^{j} \in \mathcal{G} \text { and } \mathcal{G}^{*}
\end{aligned}
$$

then for $X, Y \in$ basis of $\mathcal{G}$ :

$$
K\left(E_{i}, E_{j}\right) \equiv K_{i j}=\frac{1}{x_{a d}} f_{i m}^{n} f_{j n}^{m}
$$

where $f_{a b}{ }^{c}$ are struc. cons. of $\mathcal{G}$

The Cartan-Killing metric has many important group theoretical properties. We are interested in it because the field equations for the background fields can be viewed as if the 
level of (engineering) dimension 1 of the (generalised) Cartan-Killing metric takes its free value. To see that, we need to generalise the Cartan-Killing metric (4.2) to the case of the (inhomogenous) graded algebra (3.2). We use the direct generalisation of the expression (4.2) for the algebra (3.2) in the presence of the background fields (vielbeins). In that case the structure constants are given by (3.11). We get (the Dynkin index $x_{a d}=2$ ):

$$
K_{\mathcal{A B}}=\frac{1}{2} T_{\mathcal{A C}}{ }^{\mathcal{D}} T_{\mathcal{B D}}{ }^{\mathcal{C}}
$$

We are interested in the linearised version of previous equation. Again we expand the vielbeins to the first order and get:

$$
\begin{aligned}
& K_{\mathcal{A B}}=\frac{1}{2} f_{\mathcal{A C}}{ }^{\mathcal{D}} f_{\mathcal{B} \mathcal{D}}{ }^{\mathcal{C}}+\underbrace{\frac{1}{2} f_{\left(\mathcal{A} \mid \mathcal{C}^{\mathcal{D}} T^{(1)} \mathcal{B}\right] \mathcal{D}}{ }^{\mathcal{C}}}_{K^{(1)}{ }_{\mathcal{A B}}}+\mathcal{O}\left(E^{(2)}\right) \\
& \text { where } \quad T^{(1)}{ }_{\mathcal{A B C}}:=\frac{1}{2} D_{[\mathcal{A}} E^{(1)}{ }_{\mathcal{B C})}+\frac{1}{2} E^{(1)}\left[\mathcal{A}{ }^{\mathcal{M}} f_{\mathcal{M} \mid \mathcal{B C})}\right.
\end{aligned}
$$

\subsection{Field equations}

After imposing all the constraints we have found that everything can be expressed in terms of $E^{(1)} P \Omega$ and $E^{(1)} D \tilde{D}$. The gamma "trace" part of $E^{(1)} P \Omega$ is related directly to $E^{(1)} D \tilde{D}$ by (3.47). Therefore we want the equation of motion for the field $E^{(1)} D_{D}$.

We start with some action $S$ and vary it with respect to the vielbein $E^{D D}$ and put it to zero, i.e., $\delta / \delta E^{D D} S=0$. The variation produces a dimension 1 antisymmetric tensor. On the other hand in the previous subsection we have seen that $K_{D D}$ is the canonical antisymmetric dimension 1 tensor. Therefore we can impose the equations of motion:

$$
\frac{\delta}{\delta E^{D D}} S \equiv K_{D D}=0
$$

For the vielbein $E_{\tilde{\alpha} \beta}$ we produce the following equations:

$$
K_{\tilde{\alpha} \beta}=0 \Rightarrow K^{(1)} \tilde{\alpha}=0
$$

Plugging the definitions of structure constants and linearised torsions (note that only the combination of dimension 1 torsions is present, since lower dimensional $T^{(1)}$ torsions are all set to zero):

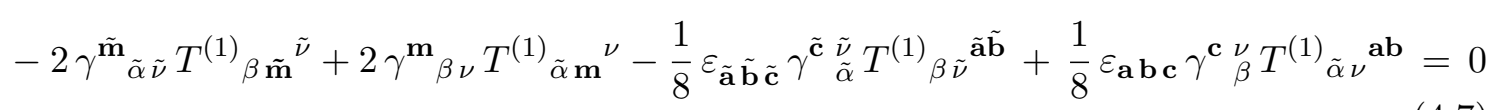

To simplify (4.7) we can use one of the linearised (super)Bianchi identities that relates $T_{\tilde{\alpha} \beta}^{(1)}{ }^{\mathbf{a b}} \equiv T^{(1)} \tilde{D} D \Sigma$ with $T^{(1)} \tilde{\alpha}^{\mathbf{a} \nu} \equiv T_{\tilde{D} P \Omega}^{(1)}$ :

$$
T^{(1)}{ }_{\tilde{\alpha} \beta}^{\mathbf{a b}}=2 \gamma_{\beta \nu}^{[\mathbf{a}} T^{(1) \mathbf{b}]} \tilde{\alpha}^{\nu}
$$

Doing that we can see that the field equation (4.7) becomes:

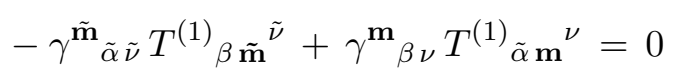


using the explicit knowledge of $T^{(1)} \tilde{\alpha} \mathbf{m}{ }^{\nu}$ from table 3 and also the result of the $\tilde{T}_{D}$ constraint (3.47). Then (4.9) can be rewritten as a differential equation just for the vielbein $E^{(1)} \tilde{D}_{D} \equiv E^{(1)} \tilde{\alpha} \beta$. For completeness we give the e.o.m. for the vielbein $E^{(1)} \tilde{\alpha}$ :

$$
\begin{gathered}
{\left[\delta_{\tilde{\alpha}}^{\tilde{\sigma}}\left(-\frac{1}{2} \gamma_{\beta}^{\mathbf{m} \nu} D_{\mathbf{m}} D^{2}-\delta_{\beta}^{\nu} \square-\gamma_{\mathbf{s} \beta}^{\nu} \cdot\left(D_{\mathbf{m}} \times D_{\mathbf{a}}\right)-2 \delta_{\beta}^{\nu} D^{\mu} D_{\mu}-2 D^{\nu} D_{\beta}\right)\right.} \\
\left.\quad+\frac{1}{2} \delta_{\beta}^{\nu} \epsilon^{\tilde{\sigma} \tilde{\epsilon}} D_{\tilde{\alpha}} \tilde{D}^{2} D_{\tilde{\epsilon}}-(\tilde{\alpha} \rightarrow \beta \text { and } \beta \rightarrow \tilde{\alpha})\right] E_{\tilde{\sigma} \nu}^{(1)}=0 \\
{\left[\left(\gamma \cdot D_{P} D_{D} \cdot D_{D}-D_{P} \cdot D_{P}-\gamma \cdot\left(D_{P} \cdot D_{P}\right)-D_{\Omega} \cdot D_{D}-D_{\Omega} D_{D}\right)\right.} \\
\left.+D_{\tilde{D}} D_{\tilde{D}} \cdot D_{\tilde{D}} D_{\tilde{D}}-(\tilde{D} \leftrightarrow D)\right] E_{\tilde{D} D}^{(1)}=0
\end{gathered}
$$

where $\square \equiv \eta^{a b} D_{a} D_{b}$ and $\gamma_{\mathbf{s} \beta}^{\nu} .\left(D_{\mathbf{m}} \times D_{\mathbf{a}}\right) \equiv \varepsilon^{\mathbf{s} \mathbf{m a}} \gamma_{\mathbf{s}}{ }_{\beta}^{\nu} D_{\mathbf{m}} D_{\mathbf{a}} \propto D_{\Sigma}$.

The remaining equations are obtained by variation of $S$ with respect to $E^{\alpha \beta}$ and $E^{\tilde{\alpha} \tilde{\beta}}$. We get:

$$
\begin{gathered}
K_{\alpha \beta}=K_{\alpha \beta}^{(1)}=0 \quad \text { and } K_{\tilde{\alpha} \tilde{\beta}}=K_{\tilde{\alpha} \tilde{\beta}}^{(1)}=0 \\
\text { where } \\
K_{\alpha \beta}^{(1)} \propto \varepsilon_{\alpha \beta} B \quad K_{\tilde{\alpha} \tilde{\beta}}^{(1)} \propto \varepsilon_{\tilde{\alpha} \tilde{\beta} \tilde{B}}
\end{gathered}
$$

Equations (4.12) and (4.13) can be rewritten in a different way:

$$
B+\tilde{B}=0 \text { and } B-\tilde{B}=0
$$

where $B$ is given by eq. (3.48). Because the explicit structure of $B$ and $\tilde{B}$ is important for the following considerations we repeat it here:

$$
\begin{aligned}
B & \propto \varepsilon^{\nu \alpha} D_{\nu}\left(D^{\tilde{\epsilon}}+\frac{1}{4} \gamma^{\tilde{\mathbf{a}} \tilde{\beta} \tilde{\epsilon}} D_{\tilde{\mathbf{a}}} D_{\tilde{\beta}}\right) E^{(1)} \tilde{\epsilon} \alpha+\varepsilon^{\alpha \beta} D_{\beta} D_{\alpha} \phi^{(1)} \\
& \equiv \varepsilon \cdot D_{D}\left(D_{\tilde{\Omega}}+\gamma \cdot D_{\tilde{P}} D_{\tilde{D}}\right) E^{(1)}{ }_{\tilde{D} D}+D_{D} \cdot D_{D} \phi^{(1)} \\
\tilde{B} & \propto \varepsilon^{\tilde{\nu} \tilde{\epsilon}} D_{\tilde{\nu}}\left(-D^{\alpha}+\frac{1}{4} \gamma^{\mathbf{a} \beta \alpha} D_{\mathbf{a}} D_{\beta}\right) E^{(1)} \tilde{\epsilon}_{\tilde{\epsilon}}+\varepsilon^{\tilde{\alpha} \tilde{\beta}} D_{\tilde{\beta}} D_{\tilde{\alpha}} \phi^{(1)} \\
& \equiv \varepsilon \cdot D_{\tilde{D}}\left(-D_{\Omega}+\gamma \cdot D_{P} D_{D}\right) E^{(1)}{ }_{\tilde{D} D}+D_{\tilde{D}} \cdot D_{\tilde{D}} \phi^{(1)}
\end{aligned}
$$

To analyse the second terms in (4.15) and (4.16) we need the following identities:

$$
\begin{aligned}
& \gamma^{\mathbf{a} \beta \alpha} D_{\mathbf{a}} D_{\beta}=4 D^{\alpha}-\frac{1}{2} D^{2} \varepsilon^{\alpha \epsilon} D_{\epsilon} \equiv D_{\Omega}-\left(D_{D} \cdot D_{D}\right) \varepsilon D_{D} \\
& \gamma^{\tilde{\mathbf{a}} \tilde{\beta} \tilde{\alpha}} D_{\tilde{\mathbf{a}}} D_{\tilde{\beta}}=-4 D^{\tilde{\alpha}}+\frac{1}{2} \tilde{D}^{2} \varepsilon^{\tilde{\alpha} \tilde{\epsilon}} D_{\tilde{\epsilon}} \equiv-D_{\tilde{\Omega}}+\left(D_{\tilde{D}} \cdot D_{\tilde{D}}\right) \varepsilon D_{\tilde{D}}
\end{aligned}
$$

where $D^{2}=\varepsilon^{\beta \alpha} D_{\alpha} D_{\beta} \equiv D_{D} \cdot D_{D}\left(\right.$ similarly for $\left.\tilde{D}^{2}\right)$.

Using (4.17) and (4.18) we get:

$$
\begin{aligned}
B & \propto-\frac{1}{8} \tilde{D}^{2}\left(\varepsilon^{\alpha \nu} \varepsilon^{\tilde{\epsilon} \tilde{\sigma}} D_{\nu} D_{\tilde{\sigma}} E^{(1)} \tilde{\epsilon} \alpha\right. \\
& \equiv-D_{\tilde{D}} \cdot D_{\tilde{D}}\left(D_{D} D_{\tilde{D}}\right) \cdot E^{(1)}{ }_{\tilde{D} D}+D_{D} \cdot D_{D} \phi^{(1)}
\end{aligned}
$$




$$
\begin{aligned}
\tilde{B} & \propto-\frac{1}{8} D^{2}\left(\varepsilon^{\alpha \nu} \varepsilon^{\tilde{\epsilon} \tilde{\sigma}} D_{\nu} D_{\tilde{\sigma}} E^{(1)}{ }_{\tilde{\epsilon} \alpha}\right)+\tilde{D}^{2} \phi^{(1)} \\
& \equiv-D_{D} \cdot D_{D}\left(D_{D} D_{\tilde{D}}\right) \cdot E^{(1)}{ }_{\tilde{D} D}+D_{\tilde{D}} \cdot D_{\tilde{D}} \phi^{(1)}
\end{aligned}
$$

Then the first of (4.14) becomes the equation:

$$
0=\left(D^{2}+\tilde{D}^{2}\right)\left(-\frac{1}{8} \varepsilon^{\alpha \nu} \varepsilon^{\tilde{\epsilon} \tilde{\sigma}} D_{\nu} D_{\tilde{\sigma}} E^{(1)} \tilde{\epsilon} \alpha+\phi^{(1)}\right)
$$

We can rewrite (4.21) using a new field $V$ :

$$
\left(D^{2}-\tilde{D}^{2}\right) V=:\left(-\frac{1}{8} \varepsilon^{\alpha \nu} \varepsilon^{\tilde{\epsilon} \tilde{\sigma}} D_{\nu} D_{\tilde{\sigma}} E^{(1)} \tilde{\epsilon} \alpha+\phi^{(1)}\right)
$$

Using this definition (4.21) can be written as:

$$
0=\left(D^{2}+\tilde{D}^{2}\right)\left(D^{2}-\tilde{D}^{2}\right) V
$$

The operator $\left(D^{2}+\tilde{D}^{2}\right)\left(D^{2}-\tilde{D}^{2}\right)$ acts on the scalar field $V$. It can be rewritten in a nicer form:

$$
\begin{aligned}
\left(D^{2}+\tilde{D}^{2}\right)\left(D^{2}-\tilde{D}^{2}\right) V & =4\left(\square-D_{\nu} D^{\nu}+D^{\nu} D_{\nu}-\left(\tilde{\square}-D_{\tilde{\nu}} D^{\tilde{\nu}}+D^{\tilde{\nu}} D_{\tilde{\nu}}\right)\right) V \\
& \equiv 4 D^{\mathcal{A}} D_{\mathcal{A}} V
\end{aligned}
$$

Therefore the first equation of (4.14) can be rewritten as:

$$
D^{\mathcal{A}} D_{\mathcal{A}} V=0
$$

\begin{tabular}{|c|c|c|}
\hline$K_{\tilde{\alpha} \beta}^{(1)}=0$ & $\Rightarrow$ & $\begin{array}{c}{\left[\delta_{\tilde{\alpha}}^{\tilde{\sigma}}\left(-\frac{1}{2} \gamma_{\beta}^{\mathbf{m} \nu} D_{\mathbf{m}} D^{2}-\delta_{\beta}^{\nu} \square-\gamma_{\mathbf{s} \beta}^{\nu} \cdot\left(D_{\mathbf{m}} \times D_{\mathbf{a}}\right)\right.\right.} \\
\left.-2 \delta_{\beta}^{\nu} D^{\mu} D_{\mu}-2 D^{\nu} D_{\beta}\right)+\frac{1}{2} \delta_{\beta}^{\nu} \epsilon^{\tilde{\sigma} \tilde{\epsilon}} D_{\tilde{\alpha}} \tilde{D}^{2} D_{\tilde{\epsilon}} \\
-(\tilde{\alpha} \rightarrow \beta \text { and } \beta \rightarrow \tilde{\alpha})] E^{(1)} \tilde{\sigma} \nu=0\end{array}$ \\
\hline$K_{\alpha \beta}^{(1)}+K_{\tilde{\alpha} \tilde{\beta}}^{(1)}=0$ & $\begin{array}{c}\Rightarrow \\
\text { where }\end{array}$ & $\begin{array}{c}D^{\mathcal{A}} D_{\mathcal{A}} V=0 \\
\left(D^{2}-\tilde{D}^{2}\right) V=:\left(-\frac{1}{8} \varepsilon^{\alpha \nu} \varepsilon^{\tilde{\epsilon} \tilde{\sigma}} D_{\nu} D_{\tilde{\sigma}} E^{(1)} \tilde{\epsilon} \alpha+\phi^{(1)}\right)\end{array}$ \\
\hline$K_{\alpha \beta}^{(1)}-K_{\tilde{\alpha} \tilde{\beta}}^{(1)}=0$ & $\Rightarrow$ & $\left(D^{2}-\tilde{D}^{2}\right)^{2} V=0$ \\
\hline
\end{tabular}

and so (4.25) is identically satisfied since it is just the strong constraint.

The second equation of (4.14) becomes the e.o.m. for the $V$ field:

$$
\left(D^{2}-\tilde{D}^{2}\right)^{2} V=0
$$

\subsection{Field equations: summary}

The field equations are summarised in the following table 4 .

Table 4. Field equations. 


\section{Dilaton}

The result of the previous section gives the structure of the linear dilaton $\phi^{(1)}$, see table 4 . Using the relation (4.22) we find the structure of the linear dilaton:

$$
\begin{aligned}
& \phi^{(1)}=\frac{1}{8} \varepsilon^{\alpha \nu} \varepsilon^{\tilde{\epsilon} \tilde{\sigma}} D_{\nu} D_{\tilde{\sigma}} E^{(1)} \tilde{\epsilon} \alpha+\left(D^{2}-\tilde{D}^{2}\right) V \\
& \equiv \varepsilon \varepsilon D_{D} D_{\tilde{D}} \cdot E_{D \tilde{D}}^{(1)}+\left(D_{D} \cdot D_{D}-D_{\tilde{D}} \cdot D_{\tilde{D}}\right) V
\end{aligned}
$$

We notice that the structure of the linear dilaton matches the structure of the dilaton field obtained by compactifying $4 \mathrm{D} \mathcal{N}=1$ supergravity to 3 dimensions, see section 7.2 .b in [17]. For the dilaton we can though impose the space-time action (after compactification of half of the dimensions, as usual in double field theory):

$$
S_{\text {dil }}:=\int d^{3} x d^{2} \theta \phi^{2}
$$

where $\phi \approx 1+\phi^{(1)}$. Moreover the cosmological constant can be added; then the action becomes:

$$
S_{\text {dil }}:=\int d^{3} x d^{2} \theta\left(\phi^{2}-\lambda V\right)
$$

\section{Conclusion}

We outline the results we have obtained: we started with T-dual $\mathcal{N}=2$ string theory, i.e,. effective $\mathcal{N}=2$ supergravity in 3 dimensions. We knew that this theory should be equivalent to the theory obtained from classical $\mathcal{N}=1$ supergravity in 4 dimensions. In this paper we first obtained the dimension -1 prepotential as the vielbein component $E^{(1)}{ }_{D \tilde{D}} \equiv E^{(1)}{ }_{\alpha \tilde{\beta}}$ and the dimension $-\frac{3}{2}$ unconstrained gauge parameter $\Lambda_{D} \equiv \Lambda_{\alpha}$ (also $\Lambda_{\tilde{D}}$ ) without solving any differential constraints. In the usual 4 dimensional $\mathcal{N}=1$ supergravity they appear only through their derivatives in objects of higher dimension after solving differential constraints, see section X.A.1 in [20]. We also derived the structure of $\mathcal{N}=2$ supergravity in 3 dimensions using the techniques of T-dually extended superspace. In particular the structure of the linear dilaton $\phi$ was derived. It matches the structure obtained from $4 \mathrm{D} \mathcal{N}=1$ and its compactification, see section 7.9 in [17] and [19]. This suggests that the T-dually extended superspace approach can be extended also to higher dimensional cases, see [7].

\section{Acknowledgments}

This work was supported in part by National Science Foundation Grant No. PHY-1316617.

Open Access. This article is distributed under the terms of the Creative Commons Attribution License (CC-BY 4.0), which permits any use, distribution and reproduction in any medium, provided the original author(s) and source are credited. 


\section{References}

[1] M. Poláček and W. Siegel, Natural curvature for manifest T-duality, JHEP 01 (2014) 026 [arXiv: 1308.6350] [INSPIRE].

[2] W. Siegel, Two vierbein formalism for string inspired axionic gravity, Phys. Rev. D 47 (1993) 5453 [hep-th/9302036] [INSPIRE].

[3] W. Siegel, Superspace duality in low-energy superstrings, Phys. Rev. D 48 (1993) 2826 [hep-th/9305073] [INSPIRE].

[4] W. Siegel, Manifest duality in low-energy superstrings, in Berkeley 1993, Proceedings, Strings ‘93, M.B. Halpern et al. eds., World Scientific, Singapore (1995) hep-th/9308133 [INSPIRE].

[5] M. Hatsuda and K. Kamimura, Classical AdS superstring mechanics, Nucl. Phys. B 611 (2001) 77 [hep-th/0106202] [INSPIRE].

[6] M. Hatsuda, Sugawara form for AdS superstring, Nucl. Phys. B 730 (2005) 364 [hep-th/0507047] [INSPIRE].

[7] M. Hatsuda, K. Kamimura and W. Siegel, Superspace with manifest T-duality from type-II superstring, arXiv: 1403.3887 [INSPIRE].

[8] C. Vafa, Evidence for F-theory, Nucl. Phys. B 469 (1996) 403 [hep-th/9602022] [InSPIRE].

[9] S.M. Kuzenko, U. Lindström and G. Tartaglino-Mazzucchelli, Off-shell supergravity-matter couplings in three dimensions, JHEP 03 (2011) 120 [arXiv:1101.4013] [INSPIRE].

[10] S.M. Kuzenko and G. Tartaglino-Mazzucchelli, Three-dimensional $N=2$ (AdS) supergravity and associated supercurrents, JHEP 12 (2011) 052 [arXiv:1109.0496] [INSPIRE].

[11] S.M. Kuzenko, U. Lindström, M. Roček, I. Sachs and G. Tartaglino-Mazzucchelli, Three-dimensional $N=2$ supergravity theories: From superspace to components, Phys. Rev. D 89 (2014) 085028 [arXiv: 1312.4267] [INSPIRE].

[12] D. Kutasov and E.J. Martinec, New principles for string/membrane unification, Nucl. Phys. B 477 (1996) 652 [hep-th/9602049] [InSPIRE].

[13] A. Achucarro, J.M. Evans, P.K. Townsend and D.L. Wiltshire, Super p-branes, Phys. Lett. B 198 (1987) 441 [INSPIRE].

[14] E. Bergshoeff, E. Sezgin and P.K. Townsend, Properties of the eleven-dimensional super membrane theory, Annals Phys. 185 (1988) 330 [INSPIRE].

[15] A. Achucarro, J.P. Gauntlett, K. Itoh and P.K. Townsend, World volume supersymmetry from space-time supersymmetry of the four-dimensional supermembrane, Nucl. Phys. B 314 (1989) 129 [inSPIRE].

[16] M.P. Blencowe and M.J. Duff, Supermembranes and the signature of space-time, Nucl. Phys. B 310 (1988) 387 [inSPIRE].

[17] S.J. Gates, M.T. Grisaru, M. Roček and W. Siegel, Superspace or one thousand and one lessons in supersymmetry, hep-th/0108200 [INSPIRE].

[18] S.J. Gates Jr., Super p form gauge superfields, Nucl. Phys. B 184 (1981) 381 [INSPIRE].

[19] M.T. Grisaru and W. Siegel, Supergraphity. Part 1. Background field formalism, Nucl. Phys. B 187 (1981) 149 [inSPIRE].

[20] W. Siegel, Fields, hep-th/9912205 [inSPIRE]. 